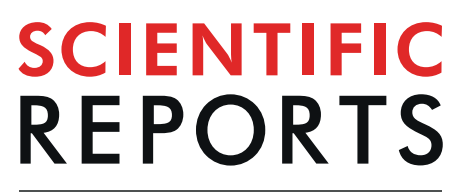

natureresearch

Corrected: Author Correction

\title{
Inhibition of translation termination by small molecules targeting ribosomal release factors
}

Xueliang Ge $\mathbb{D}^{1}$, Ana Oliveira ${ }^{1}$, Karin Hjort $\mathbb{D}^{2}$, Terese Bergfors $\mathbb{D}^{1}$, Hugo Gutiérrez-de-Terán $\left(\mathbb{1}{ }^{1}\right.$, Dan I. Andersson ${ }^{2}$, Suparna Sanyal $\mathbb{D}^{1}$ \& Johan Åqvist $\mathbb{D}^{1 *}$

The bacterial ribosome is an important drug target for antibiotics that can inhibit different stages of protein synthesis. Among the various classes of compounds that impair translation there are, however, no known small-molecule inhibitors that specifically target ribosomal release factors (RFs). The class I RFs are essential for correct termination of translation and they differ considerably between bacteria and eukaryotes, making them potential targets for inhibiting bacterial protein synthesis. We carried out virtual screening of a large compound library against 3D structures of free and ribosome-bound RFs in order to search for small molecules that could potentially inhibit termination by binding to the RFs. Here, we report identification of two such compounds which are found both to bind free RFs in solution and to inhibit peptide release on the ribosome, without affecting peptide bond formation.

Bacterial ribosomes with their auxiliary translation factors are major targets for drug intervention by a wide range of different antibiotics. Such compounds are often derived from natural products and inhibit different phases of protein synthesis, including initiation, peptide chain elongation and mRNA-tRNA translocation, or severely increase the error rate of the translation process ${ }^{1}$. Many of these antibiotics bind directly to the ribosome and thereby interfere with specific stages of protein synthesis. There are also examples, such as kirromycin and fusidic acid, which act by binding to elongation factors (EF-Tu and EF-G, respectively) and thereby blocking their release from the ribosome ${ }^{2}$. Among inhibitors that target the ribosome itself, many of them have been shown to bind either near the peptidyl transferase center (PTC), the mRNA decoding site or in the peptide exit tunnel ${ }^{1,3}$. Both the PTC, where the peptide bond formation reaction takes place, and the decoding site, where cognate aminoacyl-tRNAs are matched with the mRNA codon, are also highly conserved throughout the kingdoms of life. This makes it difficult to find molecules that selectively can block only bacterial protein synthesis. Among inhibitors of the elongation factors EF-Tu and EF-G, the only one used clinically is fusidic acid which is administered topically against skin infections ${ }^{4}$.

Interestingly, however, no specific small molecule inhibitors of translation termination have ever been reported. Termination of bacterial protein synthesis occurs when a stop codon is presented in the ribosomal A-site and is recognized by a class I release factor, RF1 or RF2. These release factors (RFs) have different but overlapping specificities, where RF1 reads UAA and UAG and RF2 reads UAA and UGA, with strong discrimination against sense codons ${ }^{5,6}$. The RFs are multi-domain proteins, where binding and stop codon recognition by domain 2 at the decoding site causes the universally conserved GGQ motif of domain 3 to insert into the A-site of the PTC, some $80 \AA$ away from the decoding site ${ }^{7}$. This event triggers hydrolysis of the peptidyl-tRNA bond in the P-site of the PTC, and the nascent peptide chain can then be released via the ribosomal exit tunnel (Fig. 1A). In eukaryotes and archaea, on the other hand a single omnipotent RF reads all three stop codons. Although the mechanism of translation termination is basically the same, there is neither sequence nor structural homology between the bacterial RFs and the eukaryotic eRF1, apart from the universally conserved GGQ motif. Hence, it could be expected that putative inhibitors of bacterial RFs and termination, may also selectively inhibit bacteria.

The only known small molecule inhibitor that affects termination is the natural product Blasticidin $\mathrm{S}$ $(\mathrm{MW}=422)$, which acts on both prokaryotic and eukaryotic cells and inhibits both peptidyl-tRNA hydrolysis and to a lesser extent peptide bond formation ${ }^{8}$. However, this drug is basically a PTC inhibitor that has been shown to deform the CCA end of the P-site RNA $^{8}$ and it binds to the ribosomal 50S subunit in a region shared

${ }^{1}$ Department of Cell and Molecular Biology, Biomedical Center, Uppsala University, SE-75124, Uppsala, Sweden. ${ }^{2}$ Department of Medical Biochemistry and Microbiology, Biomedical Center, Uppsala University, SE-75124, Uppsala, Sweden.*email: aqvist@xray.bmc.uu.se 
A

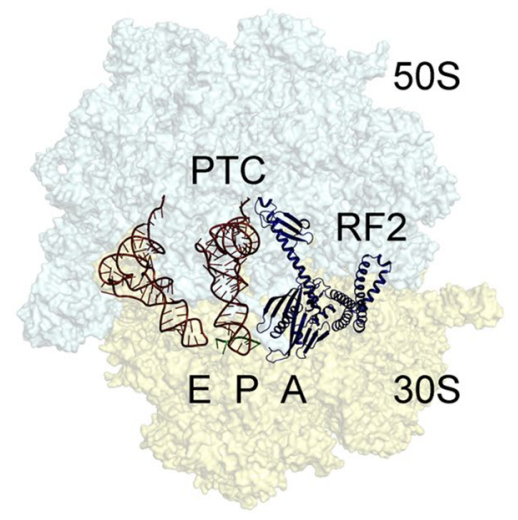

B

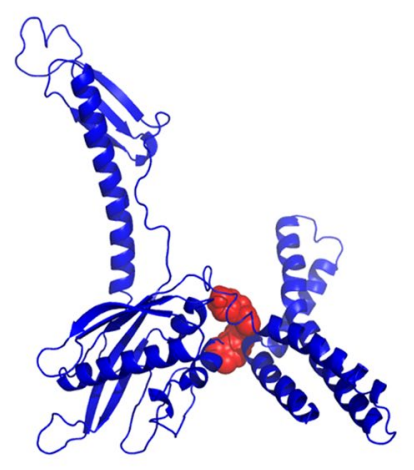

Figure 1. The bacterial 70S ribosome termination complex with RF2. (A) View of the ribosome termination complex with E- and P-site tRNAs (brown), mRNA (green) and RF2 (dark blue). (B) Close-up view of the hinge region of RF2 between domains 1 and 4 used for virtual screening, where the putative binding region is indicated by a docked ligand (red).

with several other antibiotics 9 . Hence, its mode of action is interference with the P-site tRNA rather than direct interaction with RFs. In contrast, the 18-residue antimicrobial peptide Api137 (i.e., not a small molecule) is a specific inhibitor of termination, with no effect on peptide elongation. It has recently been shown to bind in the peptide exit tunnel and establish a direct interaction with the GGQ motif of RF ${ }^{10}$. The mode of action here is particularly interesting in that Api137 was shown to enter the exit tunnel after the nascent polypeptide chain has been released, but before the RF has dissociated from the ribosome, thereby trapping the RF on it. The net effect is thus that the pool of free RF1 and RF2 is depleted, causing ribosome stalling at the stop codons ${ }^{10}$. RF1 and RF2 have very high affinity for stop codon programmed ribosomes ${ }^{11}$, with a $K_{\mathrm{d}} \sim 10^{-10} \mathrm{M}$, and their dissociation is catalyzed by the auxiliary release factor RF3. It is thus conceivable that this high intrinsic affinity could make the class I RFs vulnerable to becoming trapped on the ribosome.

We wanted to explore the possibility that small drug-like molecules might also be able to specifically inhibit translation termination by directly binding to bacterial RFs. To search for such inhibitors, we first carried out computational structure-based screening of a virtual library of commercially available compounds against 3D structures of RF2, which is the major release factor in most bacteria ${ }^{12}$. Hit molecules from this screen were then acquired and tested for their ability to $(i)$ bind to free RF2 in solution, (ii) inhibit peptide release from the ribosome in an in vitro termination assay and (iii) arrest bacterial cell growth. Herein, we identify and report two compounds with MW $\sim 440$ that fulfill these three criteria. These molecules have no effect on peptide chain elongation and are thus the first specific small molecule termination inhibitors, which may open the path for development of a new class of antibiotics.

\section{Results}

Computational analysis of RF2 as a possible drug target. The release factors RF1 and RF2 acquire an open conformation (Fig. 1A) on the $70 \mathrm{~S}$ ribosome $\mathrm{e}^{13,14}$, which is distinctly different from the closed conformation observed in crystal structures of free $\mathrm{RFs}^{15}$. The conformational equilibrium of the free RFs in solution, as revealed by SAXS experiments ${ }^{16}$, shows that this open conformation is dominating at about $80 \%$. We explored both conformations of RF2 from E. coli and T. thermophilus in search of putative binding sites with a series of molecular dynamics (MD) simulations in mixed solvents ${ }^{17}$. By using a water/ethanol solvent mixture of $80 / 20 \%$ mole fraction, this approach allows for identification of preferred hydrophobic and hydrophilic binding regions while also exploring protein flexibility. The MD simulations identified a putative druggable cavity between domains 1 and 4, approximately lined by the first and third $\alpha$-helix of domain 1 and the C-terminal $\alpha$-helix of domain 4 (Fig. 1B). This site was selected as the target for virtual screening of 3.4 million drug-like compounds from commercial libraries. These have been filtered with respect to the Lipinski rules, drug-like properties and chemical diversity to yield a structurally diverse selection of lead-like compounds. The virtual screening procedure involves molecular docking of this virtual compound library to a given $3 \mathrm{D}$ structure of the target protein. To account for protein flexibility, three representative conformations were explored in parallel by automated docking with the Glide program ${ }^{18}$. These correspond to the open ribosome-bound structure ${ }^{19,20}$, the closed crystal structure of isolated RF2 ${ }^{15,21}$ and an intermediate conformation that was found to be stable in the MD simulations. The top 200 ranked compounds were then inspected manually and 60 of them were purchased, based on their chemical diversity and repetitive pattern of interactions within the binding site (Supplementary Table 1).

Phenotypical screening of bacterial cell growth. To select molecules for further characterization of possible RF binding and termination inhibition, the 60 compounds were initially tested for their minimum inhibitory concentration (MIC) on bacterial growth. This was done using both wild type Staphylococcus aureus strain ATCC29213 $\left(\mathrm{Gram}^{+}\right)$and Escherichia coli strain MG1655 $\left(\mathrm{Gram}^{-}\right)$, for which the RF2 protein shows about $45 \%$ identity. The initial screening results are given in Table 1 for the eight compounds that were selected for further characterization of their potential interaction with the RFs. All of these $(102,115,118,128,129,137,138$ and 


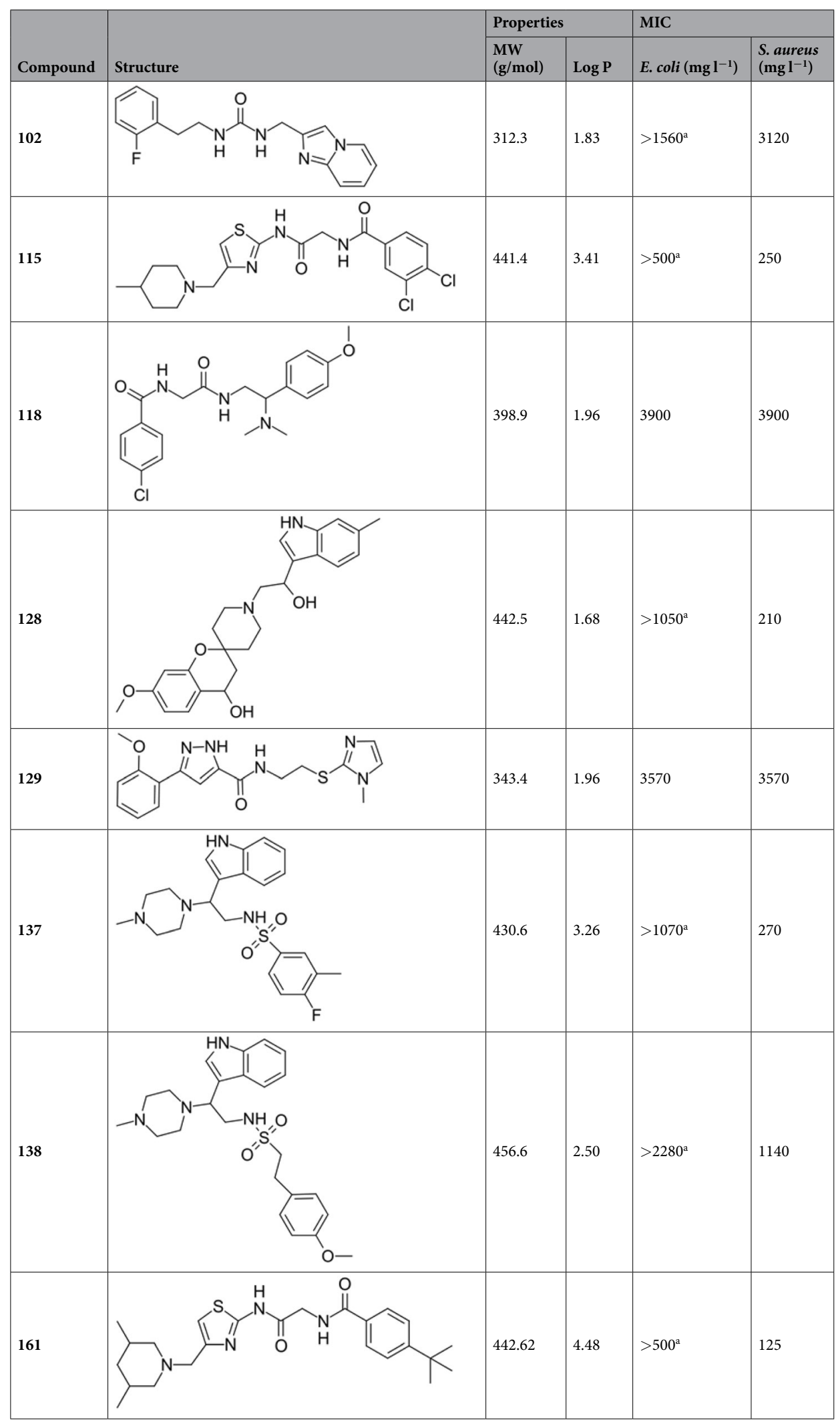

Table 1. MIC values for the initial set of compounds selected from virtual screening. ${ }^{\text {a The compound }}$ precipitated at higher concentrations in the cell culture media. 
A
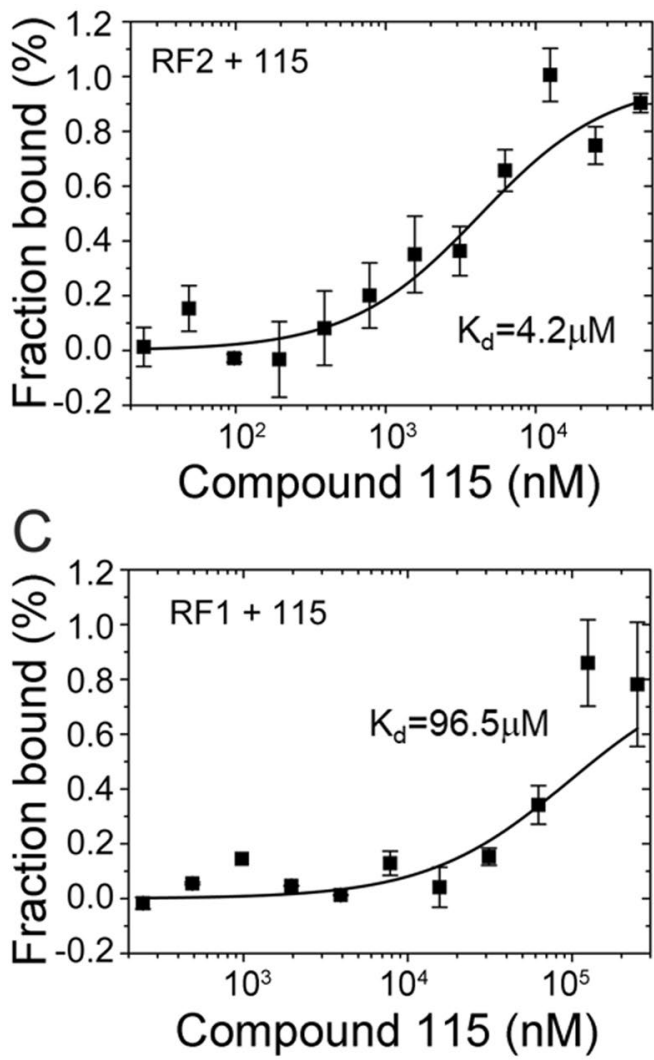

$\mathrm{B}$

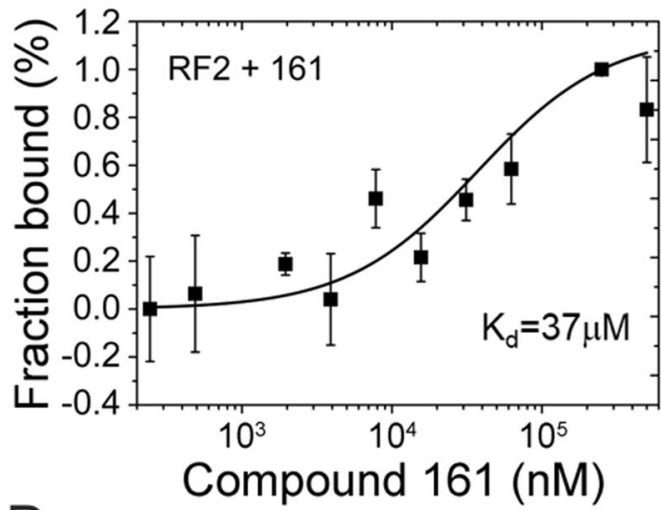

$\mathrm{D}$

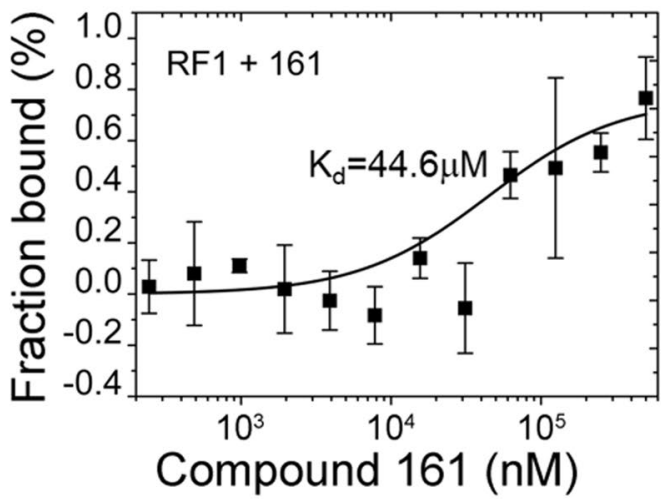

Figure 2. Binding of compounds 115 and $\mathbf{1 6 1}$ to free RF1 and RF2 in solution. Measurement of affinity between $E$. coli release factors - RF2 (A,B), RF1 (C,D) and the compounds $115(\mathbf{A}, \mathbf{C})$ and $\mathbf{1 6 1}(\mathbf{B}, \mathbf{D})$ by microscale thermophoresis. The resulting binding curves are shown (average of $\mathrm{n}=3 \pm$ s.d) from which $K_{\mathrm{d}}$ values are estimated.

161) were found to inhibit growth of $S$. aureus and two of them also prevented E. coli growth (118 and 129) at concentrations in the range of 3570 to $3900 \mathrm{mgl}^{-1}$. Each compound was analyzed at different concentrations depending on their solubility in DMSO and their propensity to precipitate in cell culture medium.

Ligand interaction with RFs in solution. To determine whether the eight selected compounds could bind to RF2 in vitro, we tested the compounds in a binding assay with free E. coli RF2 in solution. Each compound was titrated against $60 \mathrm{nM}$ of the labeled RF2 protein using microscale thermophoresis (MST). Compounds 129, 137 and 138 did not exhibit binding in the MST assay. Compounds 102, 118 and 128 on the other hand, showed precipitation at higher concentrations of DMSO than could be tolerated by the RF2 protein (15\%) and prevented us to proceed with the experiment. However, two compounds (115 and 161) changed the thermophoretic mobility and were found to bind the protein with dissociation constants $\left(K_{\mathrm{d}}\right)$ in the $\mu \mathrm{M}$ range. The $K_{\mathrm{d}}$ determined for ligands 115 and 161 were $4.2 \pm 1.7 \mu \mathrm{M}$ and $37 \pm 14 \mu \mathrm{M}$, respectively (Fig. 2A,B). These two molecules have a common scaffold, but diverge in terms of their substituents (Table 1), and both can apparently bind to free RF2 in solution. To also compare the affinity of the two best compounds to the homologous RF1 from E. coli, we performed the same assay by titrating the compounds against $10 \mathrm{nM}$ of labeled protein. Here, the $K_{\mathrm{d}}$ 's were found to be $96.5 \pm 36 \mu \mathrm{M}$ and $44.6 \pm 32 \mu \mathrm{M}$, for ligands 115 and 161, respectively (Fig. 2C,D).

The inhibitors block turnover of RF2 but do not affect peptide bond formation. The fact that the two ligands 115 and $\mathbf{1 6 1}$ evidently can bind bacterial RFs makes it particularly interesting to examine their effect in cell-free translation systems. Based on the MST results, we therefore measured inhibition of translation termination by these two compounds using a fully reconstructed in vitro translation system with purified components from $E$. coli. A peptide release complex (RC) was prepared containing mRNA programmed $70 S$ ribosomes carrying $\left[{ }^{3} \mathrm{H}\right] \mathrm{fMet}$-tRNA ${ }^{\mathrm{fMet}}$ in the P-site and a UGA stop codon in the A-site. Addition of RF2 to this mix allows release of $\left[{ }^{3} \mathrm{H}\right]$ fMet. The two compounds $\mathbf{1 1 5}$ and $\mathbf{1 6 1}$ were tested with two concentrations of RF2 and with a fixed concentration of RC. In the first single-round peptide release assay, the RF2 concentration was 10-fold higher than that of RC. Under this condition, $\left[{ }^{3} \mathrm{H}\right] \mathrm{fMet}$ from all RCs should be released at once and recycling of RF2 will not be needed. No inhibition of single-round peptide release was, however, observed even at a concentration of 
A

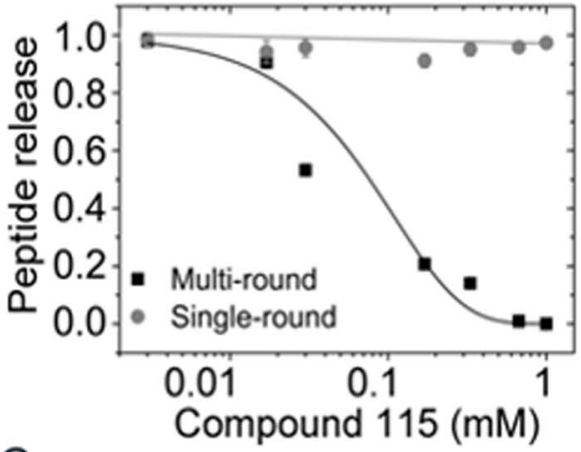

C

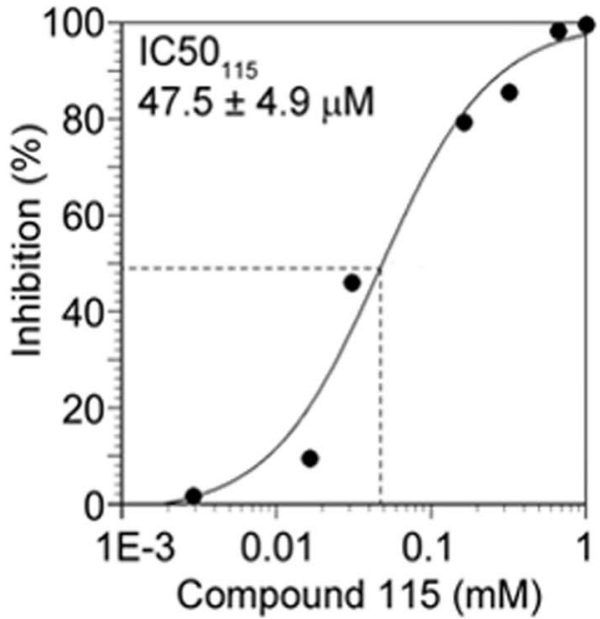

$\mathrm{E}$

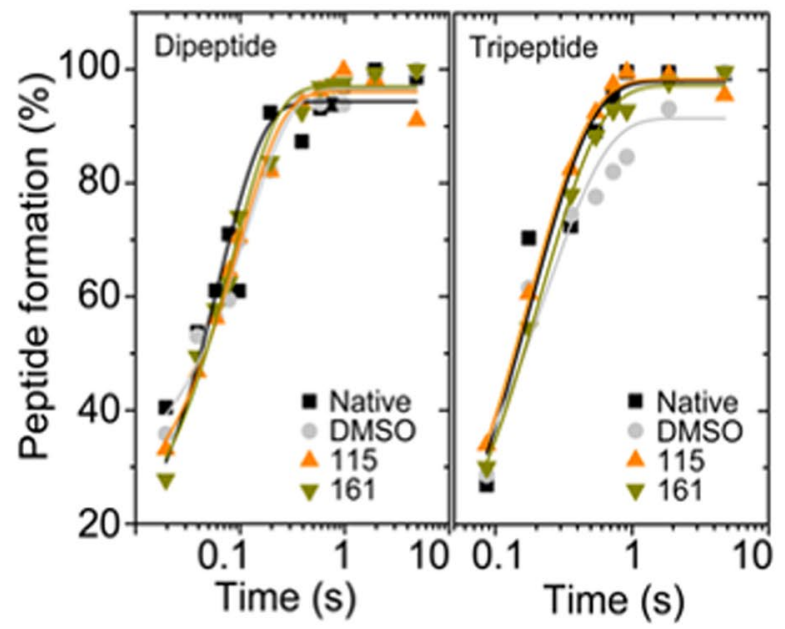

B

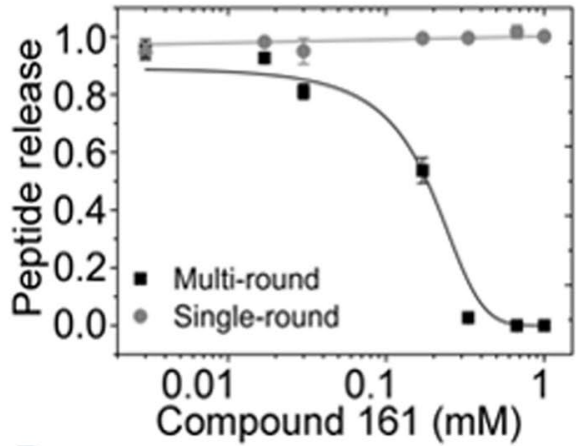

D

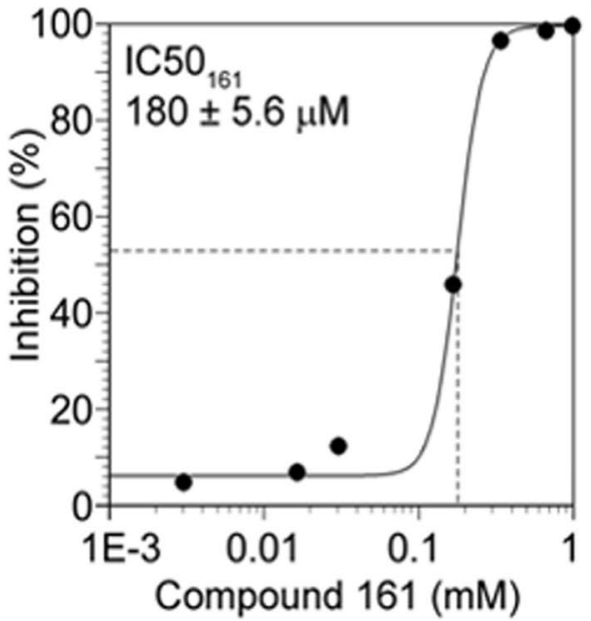

$\mathrm{F}$

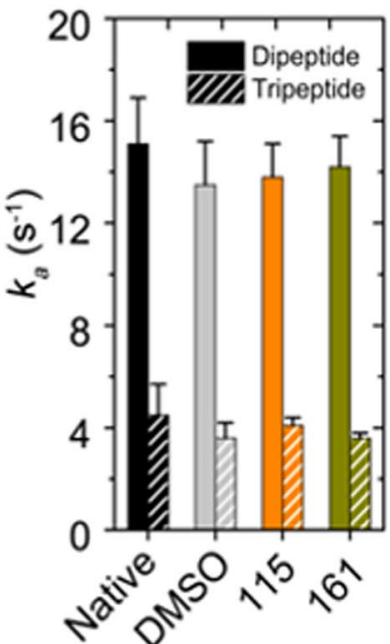

Figure 3. Effects of compounds 115 and 161 on peptide release and di- and tripeptide formation in vitro. $(\mathbf{A}, \mathbf{B})$ The effect of the compounds 115 (A) and 161 (B) on single-round (gray circle) and multiple-round (black square) peptide $\left(\left[{ }^{3} \mathrm{H}\right] \mathrm{fMet}\right)$ release assays. $(\mathbf{C}, \mathbf{D})$ Estimation of half maximal inhibitory concentration $\left(\mathrm{IC}_{50}\right)$ for the compounds $115(C)$ and $\mathbf{1 6 1}(\mathbf{D})$ from the results of the multi-round peptide release assay. (E) The effect of compounds 115 (orange) and 161 (green) at $0.5 \mathrm{mM}$ and 2\% DMSO on fMet-Leu dipeptide and fMet-Leu-Leu tripeptide formation from the quench flow experiment. Apparent rate constants $k_{a}$ for dipeptide (about $14 \mathrm{~s}^{-1}$ ) and tripeptide (about $4 \mathrm{~s}^{-1}$ ) bond formation are similar in the presence of the $\mathbf{1 1 5}$ and $\mathbf{1 1 6}$ compounds (F). All experiments were done in triplicates (average of $n=3 \pm$ s.d). 


\begin{tabular}{|l|l|l|}
\hline \multirow{2}{*}{ Strain } & \multicolumn{2}{|l|}{ MIC $\left(\mathbf{m g ~ l}^{-1}\right)$} \\
\cline { 2 - 3 } & $\mathbf{1 1 5}$ & $\mathbf{1 6 1}$ \\
\hline E. coli $\mathrm{K} 12 \mathrm{wt}$ & $>500^{\mathrm{a}}$ & $>500^{\mathrm{a}}$ \\
\hline E. coli $\Delta$ tolC & 250 & 125 \\
\hline E. coli $l p x C$ & 64 & 125 \\
\hline S. aureus $\mathrm{wt}$ & 250 & 125 \\
\hline
\end{tabular}

Table 2. MIC values for compounds 115 and 161 in different strains of E. coli and S. aureus determined by broth micro-dilution. ${ }^{a} 500 \mathrm{mg} \mathrm{l}^{-1}$ was the highest concentration tested due to precipitation in the cell culture media at higher compound concentrations.

$1 \mathrm{mM}$ of either of the two compounds (Fig. 3A,B). This indicates that neither $\mathbf{1 1 5}$ nor $\mathbf{1 6 1}$ were able to inhibit the initial association of RF2 with the RC or catalysis of the peptide release reaction.

In the second multiple-round peptide release assay, the concentration of RF2 was 50-fold lower than of that $\mathrm{RC}$, which means that multiple turnover of RF2 is required to release $\left[{ }^{3} \mathrm{H}\right] \mathrm{fMet}$ from all RC. Interestingly, in this case, inhibition of peptide release was observed (Fig. 3A,B) and the $\mathrm{IC}_{50}$ value for compound 115 was determined to be $47.5 \mu \mathrm{M}$ (Fig. $3 \mathrm{C}$ ). From this, the corresponding $K_{\mathrm{i}}$ was estimated to $3.3 \mu \mathrm{M}$, which is very close to the $K_{\mathrm{d}}$ value determined from MST experiments $\left(K_{d}=4.2 \mu \mathrm{M}\right)$. Compound 161 was found to have a higher $\mathrm{IC}_{50}$ value of $180 \mu \mathrm{M}$ (Fig. 3D), which corresponds to a $K_{\mathrm{i}}$ of $12 \mu \mathrm{M}$, again relatively close to the $K_{\mathrm{d}}$ value of $37 \mu \mathrm{M}$ obtained from the MST measurements (Fig. 2). These results strongly suggest that both molecules 115 and 161 bind to RF2 on the 70S ribosomal termination complex, thereby inhibiting dissociation and recycling of RF2, which is required for new rounds of translation. Their effect on RF1 catalyzed peptide release was also tested in the multi-round assay, but in that case no significant inhibition was observed (Supplementary Fig. 1).

In order to exclude the possibility that the two compounds might not be specific inhibitors of termination, but instead general inhibitors of the PTC, we tested them also in a di- and tripeptide formation assay ${ }^{22}$. In this experiment, a 70S initiation complex carrying an mRNA coding for Met-Leu-Leu-Stop, and $\left[{ }^{3} \mathrm{H}\right] \mathrm{fMet}-\mathrm{tRNA}^{\mathrm{fMet}}$ in the P-site was rapidly mixed at $37^{\circ} \mathrm{C}$ with an elongation mix containing the ternary complex of EF-Tu-Leu-tRNA ${ }^{\text {Leu }}$ and EF-G. The compounds 115 and 161 were premixed with the 70S ribosome at varied concentrations. As a control, the solvent DMSO was tested alone. As shown in Fig. 3E,F, no inhibition of dipeptide or tripeptide formation could be seen even at the highest concentration $(500 \mu \mathrm{M})$ of 115 and 161. This result confirms that these two compounds are not targeting ribosomes for its peptidyl transferase activity and translocation, but are specific inhibitors of RF2 turnover in the termination process.

Effect of inhibitors on cell growth. Compounds 115 and 161 were again specifically tested for their effect on bacterial growth using wt $S$. aureus, wt E. coli and the two E. coli mutants $l p x C$ and $\Delta$ tolC, where minimum inhibitory concentrations (MIC) was determined. For S. aureus, the MIC for compound 115 was $250 \mathrm{mgl}^{-1}$ $(57 \mu \mathrm{M})$ and $125 \mathrm{mgl}^{-1}(28 \mu \mathrm{M})$ for compound 161 (Table 2). For wt E. coli the MIC values of compound 115 and 161 were above the highest concentration analyzed, $500 \mathrm{mgl}^{-1}$. Higher concentrations were not possible to test for these specific compounds (115 and 161) due to their precipitation in the cell culture media above $500 \mathrm{mgl}^{-1}$. In contrast, the MICs for the E. coli drug-hypersensitive $l p x C$ mutant and the efflux-defective $\Delta$ tolC mutant were considerably lower. The $l p x C$ mutant showed MICs of $64 \mathrm{mgl}^{-1}(14 \mu \mathrm{M})$ for compound 115 and $125 \mathrm{mgl}^{-1}$ for compound 161, while the $\Delta$ tolC mutant has a MIC of $250 \mathrm{mgl}^{-1}$ for compound 115 and $125 \mathrm{mgl}^{-1}$ for compound 161. These results suggest that the outer membrane of $E$. coli is a significant barrier for uptake of the compounds, as indicated by the $l p x C$ mutant defective in lipid A biosynthesis, and that both compounds are substrates for TolC-dependent efflux. As a control, the MIC values for kirromycin were also determined for $S$. aureus and wt $E$. coli and found to be $100 \mathrm{mgl}^{-1}$ and $50 \mathrm{mgl}^{-1}$, respectively.

A time-kill assay was further used to determine if the compounds were bacteriostatic or bactericidal. Here, both compounds were found to be bactericidal, that is, the cell number decreased significantly for $S$. aureus and the tolC-defective E. coli strain during the first four hours of incubation (Fig. 4). However, after about eight hours of incubation, the number of live cells increased in all time-kill assays regardless of bacterial strain or concentration of the inhibitors $\mathbf{1 1 5}$ and $\mathbf{1 6 1}$. The reason for this regrowth is unclear, but could result from instability of the compounds, emergence of resistant mutants, or regrowth of physiologically adapted cells.

\section{Discussion}

While the ribosomal translation system is the target for many different classes of antibiotics, no small-molecule inhibitors have ever been reported that specifically target the bacterial release factors (RF1 and RF2), which are required for correct and efficient termination of protein synthesis and release of newly synthesized proteins from the ribosome. In principle, the bacterial RFs would appear as interesting drug targets, since their eukaryotic counterpart eRF1 has little sequence homology and a very different 3D structure from RF1 and RF $2^{6,13-15,23,24}$. The only known potent small-molecule inhibitor of bacterial termination is the natural product Blasticidin $S$ produced by Streptomyces bacteria, which with an apparent $K_{\mathrm{i}}$ of about $30 \mathrm{nM}$, effectively blocks peptide release ${ }^{8}$. However, this compound binds to the PTC of the ribosome ${ }^{9}$ and has been shown to deform the $3^{3}$-end of the P-site tRNA such that the tRNA conformation induces steric hindrance for RF binding to the ribosomal A-site ${ }^{8}$. The inhibitory effect of Blasticidin $S$ in termination is thus indirect and does not involve binding to the RFs themselves. Logically, therefore, the molecule also has an inhibitory effect on peptide bond formation (elongation), but with 
A

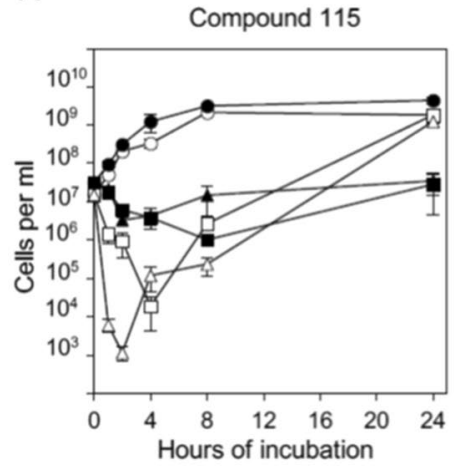

B

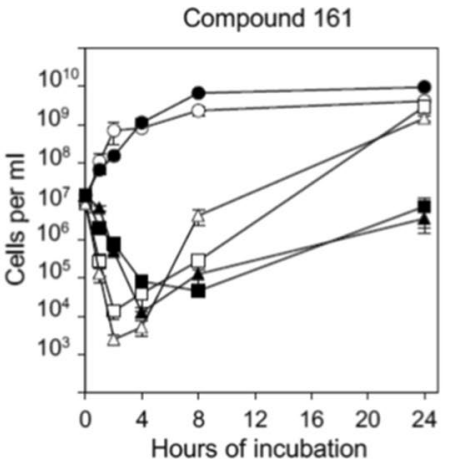

O E. coli $\Delta$ tolC

E. coli $\Delta$ tolC $1 \times$ MIC of compound

E. coli $\Delta$ tolC 2x MIC of compound

S. aureus wt

S. aureus wt $1 \times$ MIC of compound

S. aureus wt $2 \times$ MIC of compound

Figure 4. Inhibitors are bactericidal in S. aureus and tolC defective E. coli. Time-kill assays for compound 115 (A) and 161 (B) for wt S. aureus and an E. coli $\Delta t o l C$ mutant strain. Cells were incubated with or without compound 115 or 161 for 24 hours. 1x MIC (Minimal Inhibitory Concentration) of compound 115 and 161 are for E. coli and S. aureus $250 \mathrm{mgl}^{-1}$ and $125 \mathrm{mgl}^{-1}$, respectively. Samples for determination of colony forming units were withdrawn from the time-kill experiment before and 1, 2, 4, 8 and 24 hours after the compound was added. All experiments were done with triplicate biological samples (average of $n=3 \pm$ s.d).

a 6-fold higher $K_{\mathrm{i}}$ value than for the termination reaction. Moreover, it is not selective for bacteria and it also inhibits translation in mammalian cells ${ }^{25}$, which likely reflects the conserved structure of the P-site on the large ribosomal subunit.

We sought here to identify small drug-like molecules from a large virtual library with binding affinity for the free RFs in solution or bound to the ribosome, with the idea that such compounds could possibly have an effect on translation termination. The strategy was then to combine computational screening (docking) with RF binding experiments and biochemical measurements of the effects of selected compounds on translation termination in a reconstituted cell-free system. All compounds from the virtual screening that were acquired were initially screened for their effect on bacterial growth in S. aureus and E. coli strains. This was done in order to filter out a smaller subset of potentially interesting molecules for subsequent in vitro binding and inhibition experiments. Obviously, this phenotypic screening may yield both false positives and negatives with regard to the ability of the molecules to inhibit termination. However, the same would be true for initial screening of binding to the free RFs in solution since both the target site and the actual target RF conformation are unknown. The strategy employed here was, nevertheless, successful in picking out compounds that both can bind to RF2 and inhibit peptide release. The most potent compounds $\mathbf{1 1 5}$ and $\mathbf{1 6 1}$ have both binding affinities $\left(K_{\mathrm{d}}\right)$ to free RF2 and inhibition constants on the ribosome $\left(K_{\mathrm{i}}\right)$ in the multiple round peptide release assay in the low $\mu \mathrm{M}$ region. While $K_{\mathrm{i}}$ values in this range are clearly higher than those typical for tight binding drug molecules, they are unusually good for hits from a first round of virtual screening ${ }^{26}$. Moreover, neither of these molecules have any effect on peptide bond formation or tRNA translocation, which shows that they indeed are specific for termination where they most likely interact directly with the RF.

The fact that neither of the two hits $\mathbf{1 1 5}$ and $\mathbf{1 6 1}$ show any inhibition of peptide release in the single round experiments, but only under multiple round conditions when recycling of RF2 is needed, indicates that they are not able to effectively compete with RF2 binding to the ribosome. This is perhaps not so surprising considering that the affinity of the RFs for the stop codon-programmed ribosome is very high, with $K_{\mathrm{d}}$ in the sub-nM region ${ }^{11}$, compared to the $\mu \mathrm{M} K_{\mathrm{d}}$ 's measured for the small molecules. Instead it appears that the interaction of the inhibitors with the termination complex is such that RF2 becomes trapped on the ribosome and unavailable for multiple turnovers. This situation is similar to that found for the antibacterial peptide Api137 studied by Wilson, Rodnina and coworkers ${ }^{10,27}$, where binding in the exit tunnel after peptide release traps the RF on the ribosome via a direct interaction with it. It is thus likely that our inhibitors bind to and stabilize this state on the ribosome and thereby prevent conformational changes of the RFs required for their dissociation after peptide release.

It should be emphasized that the present study is not aimed at the development of new antibiotics, but rather at the (in vitro) exploration of the bacterial RFs as possible drug targets for such efforts. In particular, we were interested in exploring whether small synthetic molecules would be able to block translation termination through a mechanism involving direct binding to the RFs, which do not appear to have received much attention in antibiotics development so far. Since the results obtained from our combined computational and biochemical approach now show that small drug-like molecules can indeed inhibit termination via such a mechanism, this can be regarded as a proof-of-principle. The fact that the MIC values in the bacterial growth assays are high, as well as the actual mode of action in growth inhibition, is thus not relevant for our present purposes since these experiments were primarily used as a screening tool. A first step in further inhibitor development would rather be to try to improve in vitro affinities and inhibition by medicinal chemistry in order to arrive at intrinsically more potent compounds that could eventually be tested for effects on bacterial growth. That is, while the inhibitors discovered here are only moderately potent by normal drug standards, they could serve as useful lead compounds for exploring chemical modifications around this scaffold by organic synthesis. 


\begin{abstract}
Methods
Protein target site search and virtual screening. Crystal structures of free RF2 in the closed conformation with $\mathrm{PDB}$ codes $1 \mathrm{GQE}^{15}$ and $2 \mathrm{IHR}^{21}$, and structures of the open conformation in complex with the ribosome with $\mathrm{PDB}$ codes $5 \mathrm{DFE}^{20}$ and $4 \mathrm{~V} 9 \mathrm{~N}^{19}$, were used as input for $\mathrm{MD}$ exploration in mixed solvents with the MDmix software ${ }^{17,28}$. Each structure was immersed in a pre-equilibrated solvent box of $20 \%$ ethanol and $80 \%$ water and counter-ions were added to neutralize the system. Molecular dynamics (MD) simulations were performed with the Amber 14 program and force field using periodic boundary conditions and the particle mesh Ewald method for long-range electrostatic interactions ${ }^{29,30}$. The structures were slowly heated for $5 \mathrm{~ns}$ to the target temperature $(300 \mathrm{~K})$ in the NPT ensemble, followed by an additional $5 \mathrm{~ns}$ MD equilibration in the NVT ensemble, which was also used in the subsequent production phase. Five replicates of each system were run for $100 \mathrm{~ns}$ each, and frames were collected every 10 ps for subsequent analysis with MDmix, which estimates the probability of binding spots based on the calculated density of ethanol molecules. The output from these calculations yields a series of grids with binding free energy values for the ethanol hydroxyl (hydrophilic) and methyl (hydrophobic) probes, as well as the water occupancy.

A collection of 3.4 M compounds was kindly provided by Prof. Xavier Barril (University of Barcelona). This collection was generated from commercial libraries of six preferred vendors (Specs, Enamine, Life Chemicals, Princeton BioMolecular, Key Organics and Asinex) and further filtered to retain non-reactive and drug-like compounds. The library was prepared for docking using Schrödinger's Ligprep program ${ }^{31,32}$ and a grid of $10 \times 10 \times 10 \AA$ was built to comprise the binding site determined by the MDmix calculations. The virtual screening workflow (VSW) in the Schrödinger package (Version 15.3) was used for docking with Glide ${ }^{18}$ and final rescoring with the MM-GBSA method ${ }^{33}$. The top 200 compounds were visually inspected to yield a final selection of 60 compounds to be purchased. The tested anti-RF2 chemical compounds from the virtual screen were purchased from Enamine Ltd., Life Chemicals Inc. and Asinex Corporation (Supplementary Table 1). Ligands were dissolved in dimethyl sulfoxide (DMSO) to an initial working concentration of 5-200 mM depending on the compound.
\end{abstract}

Binding assays. A thermal shift assay using the Bio-Rad CFX Connect Real-Time PCR system was used to characterize the proteins (RF1 and RF2) and determine their stability and solubility using the JBS buffer screening kit. With this approach we determined the tolerance of the proteins against $1 \%, 5 \%, 8 \%, 10 \%$, $15 \%, 20 \%$ and $25 \%$ concentrations of DMSO. RF-ligand binding measurements were performed using the MicroScaleThermophoresis (MST) Monolith NT.115 equipment (Nano-Temper Technologies). The binding experiments were performed at $20^{\circ} \mathrm{C}$ in $0.1 \mathrm{M}$ HEPES buffer and $150 \mathrm{mM} \mathrm{NaCl}$ at $\mathrm{pH}$ 6.5. The RF2 protein was then fluorescence-labeled using the monolith Protein Labeling Kit RED-NHS (amine reactive). Twelve ligand dilutions between $500 \mathrm{nM}$ and $500 \mu \mathrm{M}$ were prepared in the same buffer as RF2. Each dilution was mixed with equal volume of $10 \mu \mathrm{l}$ of $60 \mathrm{nM}$ RF2, with a final DMSO concentration under $3 \%$. The fluorescent molecules were excited with a red laser $(650-700 \mathrm{~nm})$ in the MST instrument to monitor the spatial distribution of molecules in the capillary. Thermophoresis was measured in each capillary by locally heating the sample with an infrared laser at $40 \%$ excitation for $30 \mathrm{~s}$. Since bound and unbound molecules have a different response, the change in depletion in the presence of the inhibitor can be plotted and used to calculate the bound protein fraction. The dissociation constant $\left(\mathrm{K}_{\mathrm{d}}\right)$ was then obtained by fitting the results to a binding isotherm, using the Origin software.

Peptide release assay. The RC was formed in two steps. First E. coli 70 S ribosome $(2 \mu \mathrm{M})$, IF1, IF2 and IF3 (all $2 \mu \mathrm{M})$, XR7 Met-UGA mRNA $(10 \mu \mathrm{M})$ with sequence UAAGGAGGUAUUAAAUGUGA (Shine-Dalgarno sequence underlined; coding sequence in bold) was incubated at $37^{\circ} \mathrm{C}$ for $5 \mathrm{~min}$ in HEPES-polymix buffer at $\mathrm{pH}$ $7.5^{34}$ containing $1 \mathrm{mM}$ GTP, $1 \mathrm{mM}$ ATP, $2 \mathrm{mM}$ magnesium acetate and $0.1 \mathrm{U} / \mathrm{mL}$ Ribolock solution. Next, $2 \mu \mathrm{M}$ $\left[{ }^{3} \mathrm{H}\right]$ fMet-tRNA ${ }^{\mathrm{fMet}}$ was added to this mix and incubated for $10 \mathrm{~min}$ at $37^{\circ} \mathrm{C}$. An additional $4 \mathrm{mM}$ magnesium acetate was added to stabilize the complex. The complex was then applied to a $1.1 \mathrm{M}$ sucrose cushion (prepared in HEPES-polymix buffer $\mathrm{pH} 7.5$ ) and centrifuged at $55000 \mathrm{rpm}$ for 3 hours at $4^{\circ} \mathrm{C}$ in a S55s rotor in SORVALL M150GX ultracentrifuge. The RC pellets were washed and dissolved in HEPES-polymix buffer ( $\mathrm{pH} 7.5)$ and stored at $-80^{\circ} \mathrm{C}$ after shock freezing.

For the peptide release assay, RC and RF2 were pre-incubated for $10 \mathrm{~min}$ at $37^{\circ} \mathrm{C}$ and then mixed together in the presence or absence of the compounds 115 and 161. For the multi-round peptide release assay, $125 \mathrm{nM} \mathrm{RC}$ was mixed with $5 \mathrm{nM} \mathrm{RF} 2$ (active concentration) and the anti-RF2 compounds at $0.003-1 \mathrm{mM}$, for $5 \mathrm{~min}$ at $37^{\circ} \mathrm{C}$. For the single-round peptide release assay, $125 \mathrm{nM}$ RC was mixed with $1.250 \mu \mathrm{M}$ RF2 (active concentration) and anti-RF2 compounds $(0.003-1 \mathrm{mM})$ for 10 seconds at $37^{\circ} \mathrm{C}$. RF3 was not included in these assays and control experiments were performed in the absence of compounds and in the presence of $2 \% \mathrm{DMSO}$, which is the solvent for the anti-RF 2 compounds. The reactions were quenched by an adding equal volume of $50 \%$ formic acid. The amount of $\left[{ }^{3} \mathrm{H}\right]$ fMet released from the RC was determined by scintillation counting of the supernatant after centrifugation for 15 minutes at $14000 \mathrm{rpm}$ at $4^{\circ} \mathrm{C}$.

Tripeptide formation assay monitoring peptide bond formation and translocation. Ribosomal activity for peptide bond formation and translocation was tested by di- and tripeptide formation assays ${ }^{22}$. For that, an initiation complex and an elongation mix were first prepared in HEPES-Polymix buffer ( $\mathrm{pH} 7.5$ ). The initiation complex contained E. coli 70 S ribosome $(1 \mu \mathrm{M})$, IF1, IF2 and IF3 (all $2 \mu \mathrm{M})$, XR7 mRNA encoding Met-Leu-Leu $(10 \mu \mathrm{M})$ with sequence UAAGGAGGUAUUAAAUG CUGCUGUAA and $1 \mu \mathrm{M}\left[{ }^{3} \mathrm{H}\right]$-fMet-tRNA ${ }^{\text {fMet }}$. The elongation mix was prepared by mixing $10 \mu \mathrm{M}$ EF-Tu, $10 \mu \mathrm{M}$ EF-Ts, $10 \mu \mathrm{M}$ EF-G, $10 \mu \mathrm{M}$ tRNA ${ }^{\text {Leu }}, 0.5 \mathrm{mM}$ Leu amino acid and 1 unit of Leu-tRNA synthetase. All reactions contained $1 \mathrm{mM}$ GTP and ATP, $10 \mathrm{mM}$ phosphoenol pyruvate, $50 \mathrm{mg} / \mathrm{ml}$ pyruvate kinase and $2 \mathrm{mg} / \mathrm{ml}$ myokinase. To test the effects, $0.5 \mathrm{mM}$ of the anti-RF2 compounds 115 and 161 was added to both IC and EC together with DMSO as control. Both the mixes were incubated 
separately at $37^{\circ} \mathrm{C}$ for $15 \mathrm{~min}$. The reaction was started by mixing equal volumes of the IC and EC at $37^{\circ} \mathrm{C}$ in a quench flow apparatus and was quenched by mixing $17 \%$ formic acid after different time points. The peptides were isolated from the ribosome by $\mathrm{KOH}$ treatment and applied to reverse phase HPLC separation with in-line radioactivity detection as described earlier ${ }^{22}$.

Bacterial strains and minimum inhibitory concentration determinations. The four different strains analyzed were wild type (wt) Staphylococcus aureus (strain ATCC 29213), wt Escherichia coli (strain MG1655, K12), a drug-hypersensitive E. coli lpxC mutant D22 (strain MG1655) and an efflux-defective E. coli $\Delta$ tolC mutant (strain MG1655). These were grown in cation-adjusted Mueller Hinton II media (MH II, Nordic Biolabs), the standard medium for antibiotic susceptibility testing. For overnight cell cultures one colony from a MH II (Nordic Biolabs) plate was inoculated in $1 \mathrm{ml} \mathrm{MH} \mathrm{II} \mathrm{media} \mathrm{in} \mathrm{a} 10 \mathrm{ml}$ tube and the bacteria was incubated at $37^{\circ} \mathrm{C}$ shaking at $190 \mathrm{rpm}$. Cells from the overnight culture was used for MIC determinations and time-kill experiments.

The minimal inhibitory concentrations (MIC) of the drugs were measured in 96-well round-bottom microtiter plates using cation-adjusted Mueller-Hinton II (MH II) media. All measurements were done with at least three biological replicates. Briefly, bacterial colonies from a non-selective agar plate were re-suspended in $0.9 \% \mathrm{NaCl}$ to $0.5 \mathrm{McFarland}\left(\cong 1.5 \times 10^{8} \mathrm{cfu} / \mathrm{ml}\right)$. The cell suspension was further diluted to $\cong 5 \times 10^{5} \mathrm{cfu} / \mathrm{ml}(50 \mu \mathrm{l}$ of bacterial suspension to $10 \mathrm{ml}$ of MHII) and then $90 \mu \mathrm{l}$ of cell suspension $\left(\cong 5 \times 10^{5} \mathrm{cfu} / \mathrm{l}\right)$ was added to each well. For the initial screen of all the compounds different concentrations was used depending on the solvability of the compound in $50 \%$ DMSO and their propensity to precipitate in the cell culture media. For all compounds the MIC measurements were done in a two-fold dilution series and for compound 115 and 161 the concentration was from $500 \mathrm{mgl}^{-1}$ to $4 \mathrm{mgl}^{-1}$ by adding $10 \mu \mathrm{l}$ of compound 115 or 161 dissolved in $50 \%$ DMSO to water giving a final concentration of DMSO of $5 \%$ at the highest compound concentration $\left(500 \mathrm{mgl}^{-1}\right)$ analyzed. The positive control (without compound) also contained 5\% DMSO to determine if the DMSO concentration influenced bacterial growth. Plates were covered and incubated without shaking at $37^{\circ} \mathrm{C}$ for $16-20 \mathrm{~h}$. The MIC value was determined visually as the lowest concentration of drug that inhibited growth.

Time-kill experiments. To determine if the compounds are bacteriostatic or bactericidal, time-kill assays were performed with wt $S$. aureus and a $\Delta$ tolC efflux-defective $E$. coli. The initial inoculums were diluted from triplicate biological replicates of overnight cell cultures inoculated with one colony in $1 \mathrm{ml} \mathrm{MH} \mathrm{II} \mathrm{medium.} \mathrm{The}$ cell inoculum was diluted 1:1000 in $1 \mathrm{ml} \mathrm{MH} \mathrm{II} \mathrm{medium} \mathrm{and} \mathrm{the} \mathrm{cell} \mathrm{culture} \mathrm{was} \mathrm{allowed} \mathrm{to} \mathrm{recover} \mathrm{for} 30 \mathrm{~min}$ utes before the compounds were added at a final concentration of $1 \mathrm{x}$ MIC ( $250 \mathrm{mgl}^{-1}$ for compound 115 and $125 \mathrm{mgl}^{-1}$ for compound $\mathbf{1 6 1}$ for both bacterial strains) or 2x MIC. As a control of the possible effect of DMSO on cell growth, the positive growth controls (three biological replicates) contained the same DMSO concentration as the cells with compound added $(2 \mathrm{x}$ MIC). Samples $(20 \mu \mathrm{l})$ were withdrawn before the compound was added and also after 1, 2, 4, 8 and $24 \mathrm{~h}$ after addition. The cells were diluted in $0.9 \% \mathrm{NaCl}$ before they were plated on non-selective agar plates using glass beads. Colonies were counted after approximately $20 \mathrm{~h}$.

\section{Data availability}

The data that support the findings of this study are available from the corresponding author upon reasonable request.

Received: 19 June 2019; Accepted: 10 October 2019;

Published online: 28 October 2019

\section{References}

1. Wilson, D. N. Ribosome-targeting antibiotics and mechanisms of bacterial resistance. Nat. Rev. Microbiol. 12, 35-48 (2014).

2. Borg, A. et al. Fusidic acid targets elongation factor G in several stages of translocation on the bacterial ribosome. J. Biol. Chem. 290, 3440-3454 (2015).

3. Poehlsgaard, J. \& Douthwaite, S. The bacterial ribosome as a target for antibiotics. Nat. Rev. Microbiol. 3, 870-881 (2005).

4. Williamson, D. A., Carter, G. P. \& Howden, B. P. Current and emerging topical antibacterials and antiseptics: agents, action and resistance patterns. Clin. Microbiol. Rev. 30, 827-860 (2017).

5. Freistroffer, D. V., Kwiatkowski, M., Buckingham, R. H. \& Ehrenberg, M. The accuracy of codon recognition by polypeptide release factors. Proc. Natl. Acad. Sci. USA 97, 2046-2051 (2000).

6. Zaher, H. S. \& Green, R. Fidelity at the molecular level: lessons from protein synthesis. Cell 136, 746-762 (2009).

7. Youngman, E. M., McDonald, M. E. \& Green, R. Peptide release on the ribosome: mechanism and implications for translational control. Ann. Rev. Microbiol. 62, 353-373 (2008).

8. Svidritskiy, E., Ling, C., Ermolenko, D. N. \& Korostelev, A. A. Blasticidin S inhibits translation by trapping deformed tRNA on the ribosome. Proc. Natl. Acad. Sci. USA 110, 12283-12288 (2013).

9. Hansen, J. L., Moore, P. B. \& Steitz, T. A. Structures of five antibiotics bound at the peptidyl transferase center of the large ribsomal subunit. J. Mol. Biol. 330, 1061-1075 (2003).

10. Florin, T. et al. An antimicrobial peptide that inhibits translation by trapping release factors on the ribosome. Nat. Struct. Mol. Biol. 24, 752-757 (2017).

11. Zavialov, A. V., Mora, L., Buckinghan, R. H. \& Ehrenberg, M. Release of peptide promoted by the GGQ motif of class 1 release factors regulates the GTPase activity of RF3. Mol. Cell 10, 789-798 (2002).

12. Adamski, F. M., Mccaughan, K. K., Jorgensen, F., Kurland, C. G. \& Tate, W. P. The concentration of polypeptide-chain release factor-1 and factor-2 at different growth-rates of Escherichia coli. J. Mol. Biol. 238, 302-308 (1994).

13. Laurberg, M. et al. Structural basis for translation termination on the 70S ribosome. Nature 454, 852-857 (2008).

14. Weixlbaumer, A. et al. Insights into translational termination from the structure of RF2 bound to the ribosome. Science 322, $953-956$ (2008).

15. Vestergaard, B. et al. Bacterial polypeptide release factor RF2 is structurally distinct from eukaryotic eRF1. Mol. Cell 8, 1375-1382 (2001). 
16. Vestergaard, B. et al. The SAXS solution structure of RF1 differs from its crystal structure and is similar to its ribosome bound cryoEM structure. Mol. Cell 20, 929-938 (2005).

17. Alvarez-Garcia, D. \& Barril, X. Molecular simulations with solvent competition quantify water displaceability and provide accurate interaction maps of protein binding sites. J. Med. Chem. 57, 8530-8539 (2014).

18. Friesner, R. A. et al. Glide: a new approach for rapid, accurate docking and scoring. 1. method and assessment of docking accuracy. J. Med. Chem. 47, 1739-1749 (2004).

19. Santos, N., Zhu, J., Donohue, J. P., Korostelev, A. A. \& Noller, H. F. Crystal structure of the 70S ribosome bound with the Q253P mutant form of release factor RF2. Structure 21, 1258-1263 (2013).

20. Pierson, W. E. et al. Uniformity of peptide release is maintained by methylation of release factors. Cell Rep. 17, 11-18 (2016).

21. Zoldak, G. et al. Release Factors 2 from Escherichia coli and Thermus thermophilus: structural, spectroscopic and microcalorimetric studies. Nucl. Acids Res. 35, 1343-1353 (2007).

22. Holm, M., Borg, A., Ehrenberg, M. \& Sanyal, S. Molecular mechanism of viomycin inhibition of peptide elongation in bacteria. Proc. Natl. Acad. Sci. USA 113, 978-983 (2016).

23. Song, H. et al. The crystal structure of human eucaryotic release factor eRF1-mechanism of stop codon recognition and peptidyltRNA hydrolysis. Cell 100, 311-321 (2000).

24. Shao, S. et al. Decoding mammalian ribosome-mRNA states by translational GTPase complexes. Cell 167, 1229-1240 (2016).

25. Yamaguchi, H., Yamamoto, C. \& Tanaka, N. Inhibition of protein synthesis by Blasticidin S. J. Biol. Chem. 57, 667-677 (1965).

26. Luzhkov, V. B. et al. Virtual screening and bioassay study of novel inhibitors for the dengue virus mRNA capo (nucleoside-2'O)methyltransferase. Bioorg. Med. Chem. 15, 7795-7802 (2007).

27. Graf, M. et al. Visualization of translation termination intermediates trapped by the Apidaecin 137 peptide during RF3-mediated recycling of RF1. Nat. Comm. 9, 3053 (2018).

28. Seco, J., Luque, F. J. \& Barril, X. Binding site detection and druggability index from first principles. J. Med. Chem. 52, 2363-2371 (2009).

29. Case, D. A. et al. The Amber biomolecular simulation programs. J. Comput. Chem. 26, 1668-1688 (2005).

30. Maier, J. A. et al. ff14SB: improving the accuracy of protein side chain and backbone parameters from ff99sb. J. Chem. Theory Comput. 11, 3696-3713 (2015).

31. Shelley, J. C. et al. Epik: a software program for $\mathrm{pK}$ a prediction and protonation state generation for drug-like molecules. J. Comput.Aided Mol. Design 21, 681-691 (2007).

32. Greenwood, J. R., Calkins, D., Sullivan, A. P. \& Shelley, J. C. Towards the comprehensive, rapid, and accurate prediction of the favorable tautomeric states of drug-like molecules in aqueous solution. J. Comput.-Aided Mol. Design 24, 591-604 (2010).

33. Li, J. et al. The VSGB 2.0 model: a next generation energy model for high resolution protein structure modeling. Proteins 79, 2794-2812 (2011).

34. Koripella, R. K. et al. A conserved histidine in switch-II of EF-G moderates release of inorganic phosphate. Sci. Rep. 5, 12970 (2015).

\section{Acknowledgements}

We thank Prof. Xavier Barril for the chemical library used to perform virtual screening and Dr. Annette Roos for expert technical support. This work was funded by the Swedish Research Council (VR) and the Knut and Alice Wallenberg Foundation. Support from the Swedish National Infrastructure for Computing (SNIC) is also gratefully acknowledged. Open access funding provided by Uppsala University.

\section{Author contributions}

X.G. performed peptide release and peptide formation experiments. A.O. performed virtual screening and binding experiments. K.H. conducted bacterial growth experiments. T.B. assisted with binding experiments. X.G., A.O., K.H., T.B., H.G.T., D.A., S.S. and J.Å. analyzed data and wrote the paper. J.Å. designed research.

\section{Competing interests}

The authors declare no competing interests.

\section{Additional information}

Supplementary information is available for this paper at https://doi.org/10.1038/s41598-019-51977-1.

Correspondence and requests for materials should be addressed to J.Å.

Reprints and permissions information is available at www.nature.com/reprints.

Publisher's note Springer Nature remains neutral with regard to jurisdictional claims in published maps and institutional affiliations.

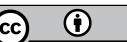

Open Access This article is licensed under a Creative Commons Attribution 4.0 International License, which permits use, sharing, adaptation, distribution and reproduction in any medium or format, as long as you give appropriate credit to the original author(s) and the source, provide a link to the Creative Commons license, and indicate if changes were made. The images or other third party material in this article are included in the article's Creative Commons license, unless indicated otherwise in a credit line to the material. If material is not included in the article's Creative Commons license and your intended use is not permitted by statutory regulation or exceeds the permitted use, you will need to obtain permission directly from the copyright holder. To view a copy of this license, visit http://creativecommons.org/licenses/by/4.0/.

(C) The Author(s) 2019 